\title{
Scaling Properties of the Two-Dimensional Randomly Stirred Navier-Stokes Equation
}

\author{
Andrea Mazzino* \\ Department of Physics, University of Genova, and INFN, CNISM, Genova Section, Via Dodecaneso 33, I-16146 Genova, Italy \\ Paolo Muratore-Ginanneschi ${ }^{\dagger}$ \\ Department of Mathematics and Statistics, University of Helsinki, P.O. Box 4, 00014 Helsinki, Finland \\ Stefano Musacchio \\ Department of Physics of Complex Systems, Weizmann Institute of Science, Rehovot 76100, Israel
}

(Received 8 January 2007; published 4 October 2007)

\begin{abstract}
We inquire into the scaling properties of the 2D Navier-Stokes equation sustained by a force field with Gaussian statistics, white noise in time, and with a power-law correlation in momentum space of degree $2-2 \varepsilon$. This is at variance with the setting usually assumed to derive Kraichnan's classical theory. We contrast accurate numerical experiments with the different predictions provided for the small $\varepsilon$ regime by Kraichnan's double cascade theory and by renormalization group analysis. We give clear evidence that for all $\varepsilon$, Kraichnan's theory is consistent with the observed phenomenology. Our results call for a revision in the renormalization group analysis of (2D) fully developed turbulence.
\end{abstract}

PACS numbers: 47.27.ef, 47.27.ek

Strong experimental and numerical evidence in 3D turbulence (see, e.g., [1,2]) indicates the independence of the injection mechanism in the limit of high Reynolds numbers. This is fully consistent with the scenario in the manner of Kolmogorov for 3D turbulence [1,3] exhibiting a universal scaling regime in the so-called inertial range of scales. A first-principles theory of fully developed turbulence is, however, missing and represents an highly desirable goal. Attempts to investigate from first principles the dependence of scaling behavior on the injection mechanisms have been accomplished in [4,5] (see [6] for a review) by applying a renormalization group improved perturbation theory (RG) to the 3D randomly stirred Navier-Stokes equation with power law forcing. Recent numerical experiments [7-9] corroborated the scenario predicted by RG within numerical accuracy.

In many situations the flow of a shallow layer of fluid can be described by the 2D incompressible Navier-Stokes equation [10]. Although in 2D the simultaneous conservation of energy and enstrophy allows one to rigorously settle issues of existence and uniqueness of solutions both for the deterministic Cauchy problem [11] and, very recently, for the stochastically forced [12-15] Navier-Stokes equation, the understanding of universality for large Reynolds is limited to a phenomenological theory due to Kraichnan [16] and Batchelor [17] (see also [18] for a review). Kraichnan's theory predicts the presence of a double cascade mechanism governing the transfer of energy and enstrophy in the limit of infinite inertial range. Accordingly, an inverse energy cascade with spectrum characterized by a scaling exponent $d_{E}=-5 / 3$ appears for values of the wave number $k$ smaller than the typical scale $k_{F}$ of the forcing scale. For wave numbers larger than $k_{F}$ a direct enstrophy cascade should occur. The corresponding energy spectrum scales as $d_{E}=-3+\ldots$ where the dots here stand for possible logarithmic corrections. As emphasized in [19] Kraichnan's theory is encoded in three hypotheses: (i) that velocity correlations be smooth at finite viscosity and exist in the inviscid limit even at coinciding points, (ii) that Galilean invariant functions and, in particular, structure functions reach a steady state, and (iii) that no dissipative anomalies occur for the energy cascade. Very strong laboratory evidences (see [20,21] for a review) dealing with electromagnetically driven liquid metals [22] and soap films [23] together with numerical simulations (see, e.g., [24] and references therein) support Kraichnan's theory. In 2D, the application of RG techniques to the Navier-Stokes equation gives rise to extra difficulties [25] and only recently a self-consistent approach has been introduced [26,27]. Surprisingly, at variance with the $3 \mathrm{D}$ case, RG predictions lead to a scenario which appears at odds with the phenomenological available (Kraichnan's) theory. The goal of the present work is to shed light on this issue.

The RG starting point is the randomly stirred NavierStokes equation

$$
\begin{aligned}
\left(\partial_{t}+v^{\beta} \partial_{\beta}\right) v^{\alpha}-\nu_{0} \partial^{2} v^{\alpha} & =-\partial^{\alpha} P+f^{\alpha}-\xi_{0} v^{\alpha} \\
\partial^{\beta} v_{\beta} & =0
\end{aligned}
$$

where we adopted Einstein convention for repeated indexes, $\alpha, \beta=1,2, P$ is the pressure enforcing incompressibility and $\xi_{0}$ is an Ekman type coupling providing for large scale dissipation. The forcing $\mathbf{f}$ is a Gaussian field with zero average and correlation

$$
\begin{gathered}
\prec f^{\alpha}(\mathbf{x}, t) f^{\beta}(\mathbf{y}, s) \succ=\delta(t-s) F^{\alpha \beta}(\mathbf{x}-\mathbf{y}) \\
F^{\alpha \beta}(\mathbf{x}):=\int \frac{d^{d} p}{(2 \pi)^{d}} e^{i \mathbf{p} \cdot \mathbf{x}} \check{F}(p) T^{\alpha \beta}(\hat{\mathbf{p}})
\end{gathered}
$$




$$
\check{F}(p):=\frac{F_{0} \chi\left(\frac{p}{m}, \frac{p}{M}\right)}{p^{d-4+2 \varepsilon}}
$$

$F_{0}$ is a constant specifying the amplitude of forcing fluctuations and $T^{\alpha \beta}(\hat{\mathbf{p}})$ is the transversal projector. The function $\chi$ in (4) is slowly varying for $m \ll p \ll M$ and set infrared $m$ and ultraviolet $M$ cutoffs for the forcing. Its detailed shape does not affect the RG scaling predictions. Irrespectively of the spatial dimension $d$, the cumulative spectrum $F_{\alpha}^{\alpha}(0)$ of the forcing diverges for $0 \leq \varepsilon<2$ as the ultraviolet cutoff $M$ tends to infinity, hence providing for stirring at small spatial scales. For $\varepsilon>2, F_{\alpha}^{\alpha}(0)$ is dominated by small wave numbers and thus describes infrared stirring. At $\varepsilon=0$, the scaling dimensions of the material derivative, dissipation, and forcing in (1) coincide for a scaling dimension of the velocity field $d_{v}=1$ in momentum units. Thus $\varepsilon=0$ provides a marginal limit around which it should be possible to determine perturbatively scaling dimensions by means of ultraviolet RG. The main result $[26,27]$ is the existence of a non-Gaussian infrared stable fixed point of the RG flow yielding for the energy spectrum the prediction

$$
E(k)=\varepsilon^{1 / 3} F_{0}^{2 / 3} k^{1-(4 \varepsilon / 3)} R\left(\varepsilon, \frac{m}{k}, \frac{k_{b}}{k}\right)
$$

The adimensional function $R$ depends upon infrared scales $m$ and $k_{b}=\left(\varepsilon \xi_{0}^{3} / F_{0}\right)^{1 /(6-2 \varepsilon)}$ and admits a regular expansion in powers of $\varepsilon$ at the RG fixed point [26]. The resummation leading to $k^{1-(4 \varepsilon / 3)}$ in (5) is derived from the solution of a Callan-Symanzik equation [28] which in the present case reduces to matching the scaling dimensions of the two terms in the material derivative with the forcing's one, i.e., to the requirement of Galilean invariance alone. According to $[6,26]$ composite operators at small $\varepsilon$ do not induce any self-similarity breaking by the infrared scales $m$ and $k_{b}$ so that these latter can be set to zero in (5). The conclusion is that the spectrum should scale for small $\varepsilon$ with exponent $d_{E}=1-4 \varepsilon / 3$ as in the $3 \mathrm{D}$ case $[5,6]$. In $3 \mathrm{D}$ the $\varepsilon$ dependence of the spectrum is consistent with physical intuition. For small $\varepsilon$ stirring describes thermal fluctuations while a turbulent regime with $d_{E}=-5 / 3$ Kolmogorov's spectrum are expected to occur only at $\varepsilon=2$ (see Chap. 1.8 of [6] for a discussion of $\varepsilon>2$ ). Such a scenario has been verified within numerical accuracy in [7-9]. In 2D the result seems at odds with the idea of inverse energy cascade. Namely under assumptions (i), (ii), (iii) $[19,29,30]$ the Kármán-Howarth-Monin equation stemming from (1)-(4)

$$
\begin{aligned}
\left(\partial_{t}+\xi_{0}-\right. & \left.\nu_{0} \partial^{2}\right) \prec v^{\alpha}(\mathbf{x}, t) v_{\alpha}(0, t) \succ \\
& -\frac{1}{2} \partial_{\mu} \prec\|\delta v\|^{2}(\mathbf{x}, t) \delta v^{\mu}(\mathbf{x}, t) \succ=F^{\alpha}{ }_{\alpha}(\mathbf{x})
\end{aligned}
$$

with $\delta v^{\alpha}(\mathbf{x}, t)=v^{\alpha}(\mathbf{x}, t)-v^{\alpha}(0, t)$ yields for $\xi_{0}=0$, $\varepsilon<2$

$$
S_{3}(x) \simeq c_{1} F_{0} M^{4-2 \varepsilon} x+\frac{c_{2} F_{0}}{x^{3-2 \varepsilon}}, \quad m^{-1} \gg x \gg M^{-1}
$$

$S_{3}$ denotes the three point velocity longitudinal structure function and $c_{i} i=1,2$ two adimensional coefficients irrelevant for the present argument. Power counting based on (7) then predicts a $d_{E}=-5 / 3$ inverse cascade spectrum at small $\varepsilon$ with at most subleading corrections consistent with the RG prediction.

It is worthwhile to contrast the two apparently discordant predictions (5) and (7) with the actual Navier-Stokes phenomenology. To attain this goal we integrated the Navier-Stokes equation (1) for the vorticity field $(\omega=$ $\nabla \times \mathbf{v}$ ) with a fully dealiased pseudospectral method [31] in a doubly periodic square domain of size $L=2 \pi$ at resolution $N^{2}=1024^{2}$ and $2048^{2}$. Dealiasing cutoff is set to $k_{t}=N / 3$. Time evolution was computed by means of a second-order Runge-Kutta scheme, with implicit handling of the linear friction and viscous terms. As customary (see, e.g., [32,33]) we added to (1) a hyperviscous damping $(-1)^{p-1} \nu_{p-1} \partial^{2 p} v$ [34]. The integration time has been carried out for 20 large eddy turnover times after the velocity fields have reached the stationary state. The stochastic forcing is implemented in Fourier space by means of Gaussian, white-in-time noise as in [35] but with variance determined according to (4). Parameters of the simulations are reported in the figure captions.

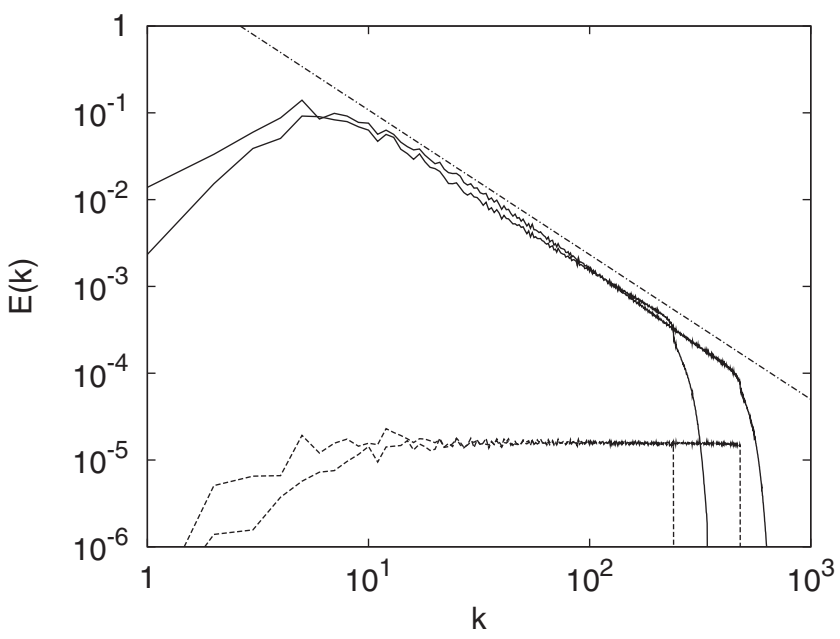

FIG. 1. Energy spectrum for $\varepsilon=0.5$. An inertial range with inverse energy cascade sets in for sufficiently small viscosity (solid line). At larger viscosity the inertial range is suppressed and dissipative spectrum $E(k) \sim k^{1-2 \varepsilon}$ is observed (dashed line). Parameters values are $m=1, M=240, \nu_{0}=10^{-4}, \nu_{7}=$ $10^{-38}, \xi_{0}=0.1, F_{0}=4 \times 10^{-4}$ and $m=1, M=240, \nu_{0}=$ $10^{-3}, \nu_{7}=10^{-38}, \xi_{0}=4 \times 10^{-3}, F_{0}=4 \times 10^{-4}$ for the simulations at resolution $N^{2}=1024^{2}$ and $m=1, M=480, \nu_{0}=$ $10^{-5}, \nu_{7}=10^{-42}, \xi_{0}=0.14, F_{0}=10^{-4}$, and $m=1, M=$ 480, $\nu_{0}=10^{-3}, \nu_{7}=10^{-42}, \xi_{0}=10^{-2}, F_{0}=10^{-4}$ for the simulations at resolution $N^{2}=2048^{2}$. 


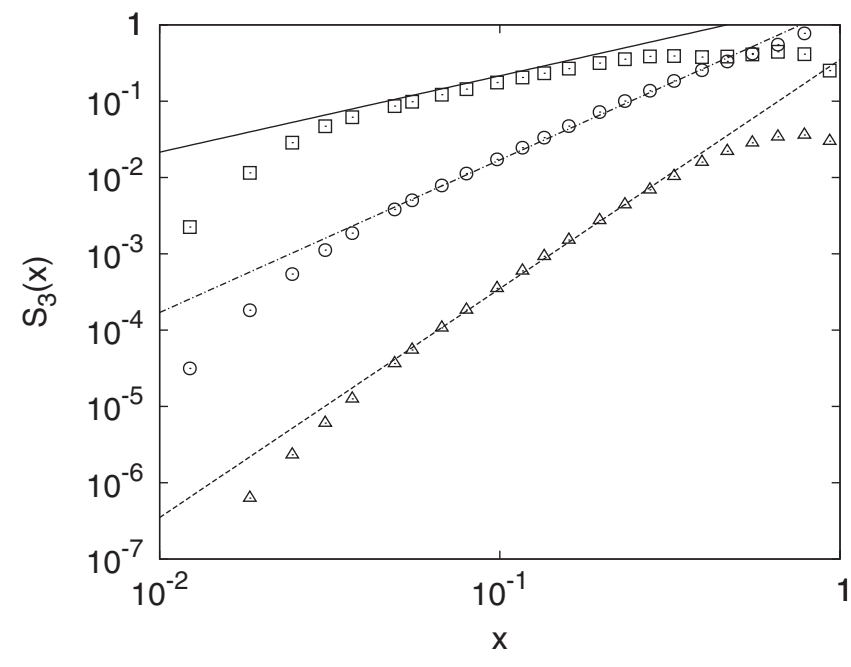

FIG. 2. Third order structure function of longitudinal velocity increments $S_{3}(x)$ for $\varepsilon=1$ (squares), $\varepsilon=2.5$ (circles), $\varepsilon=4$ (triangles). When $\varepsilon<2$ the presence of an inverse energy cascade with constant flux results in the scaling $S_{3}(x) \sim x$ (solid line). When $\varepsilon>3$ the presence of a direct enstrophy cascade with constant flux results in the scaling $S_{3}(x) \sim x^{3}$ (dashed line). For $2<\varepsilon<3$ the scaling is compatible with local balance (dash-dotted line). Parameters values are $N=1024^{2}, \xi_{0}=$ $0.1, \nu_{0}=0, \nu_{7}=10^{-18}, F_{0}=4 \times 10^{-4}$, for $\varepsilon=1 ; \xi_{2}=4$, $\nu_{0}=0, \nu_{7}=10^{-18}, F_{0}=10^{4}$, for $\varepsilon=2.5$, and $\xi_{1}=2, \nu_{0}=$ $0, \nu_{7}=10^{-18}, F_{0}=10^{4}$, for $\varepsilon=4$. The forcing cutoffs are set to $m=6$ and $M=240$.

We repeated our numerical experiments for different values of $\varepsilon$. The outcomes evince that for all $0 \leq \varepsilon \leq 2$ and sufficiently small viscosity, an inverse energy cascade with exponent consistent with $d_{E}=-5 / 3$ is observed (see Fig. 1). At scales larger than those in the inertial range, the spectrum behavior is compatible with the one given by the balance between forcing and the Ekman term. If the viscosity increases, finite resolution effects prevent the observation of a clean inertial range. The corresponding spectra are in any case largely incompatible with the RG prediction. Finally, as the viscous dissipative scale becomes of the order of the infrared cutoff scale a spectrum appears with scaling exponent consistent with the prediction $d_{E}=$ $1-2 \varepsilon$ dictated by the balance between forcing and dissipation (see again Fig. 1). As shown in Fig. 1 these results are not dependent on the value of the ultraviolet cutoff $M$, and no crossover to an RG scaling region is observed.

Local balance scaling becomes dominant in the range $2<\varepsilon<3$ (see Fig. 2). There, energy spectra scale with an exponent compatible with $d_{E}=1-4 \varepsilon / 3$. Finally, for $\varepsilon>$ 3 a direct enstrophy cascade invades the whole inertial range. Accurate numerical evidence corroborated by phenomenological arguments [32,33,35] upholds nonuniversal dependence of the kinetic energy spectrum upon the Ekman friction. Logarithmic corrections to the spectrum at $\xi_{0}=0$ should be then interpreted as the signature of marginality [32]. Since the aim of our numerics at $\varepsilon>2$ was to identify the energy and enstrophy inputs, respectively, at $I_{E}\left(k, k_{0}\right) \propto \int_{k_{0}}^{k} d q q \check{F}(q)$ and $I_{Z}\left(k, k_{0}\right) \propto$ $\int_{k_{0}}^{k} d q q^{3} \check{F}(q)\left(k_{0}\right.$ is a suitable integration constant, see caption of Fig. 3), as relevant order parameters for the steady state, we replaced for $\varepsilon>2$ the Ekman friction with a hypodissipative term $(-1)^{q+1} \xi_{q} \partial^{-2 q} v$ which is expected to suppress the aforementioned nonuniversal corrections to the spectrum [36].

The existence of three distinct regimes versus $\varepsilon$ is confirmed by the inspection of the energy and enstrophy fluxes in the inertial range (see Fig. 3). These latter are, respectively, $\quad \Pi_{E}\left(k, k_{0}\right) \propto \int_{k_{0}}^{k} \frac{d^{2} q}{(2 \pi)^{2}} \Re \prec \mathcal{F}\left\{\boldsymbol{v}^{\alpha}\right\} \times$ $(-q) \mathcal{F}\left\{\left(v^{\beta} \partial_{\beta} v_{\alpha}\right)\right\}(q) \succ, \quad \Pi_{Z}\left(k, k_{0}\right) \propto \int_{k_{0}}^{k} \frac{d^{2} q}{(2 \pi)^{2}} \Re \prec \mathcal{F}\{\omega\} \times$ $(-q) \mathcal{F}\left\{\left(v^{\beta} \partial_{\beta} \omega\right)\right\}(q) \succ$ where $\mathcal{F}$ denotes the Fourier transform while $k_{0}$ and the sign of the proportionality factor are chosen such that $\Pi_{E}$ and $\Pi_{Z}$ are positive functions of $k$ in the inertial range.

For $0 \leq \varepsilon<2$ both $I_{E}$ and $I_{Z}$ concentrate in the ultraviolet. Since stirring occurs mainly at small spatial scales an inverse energy cascade sets in the whole inertial range [Fig. 3(a)]. In the range $2<\varepsilon<3$ the energy input becomes infrared divergent while $I_{Z}$ is still ultraviolet diver-
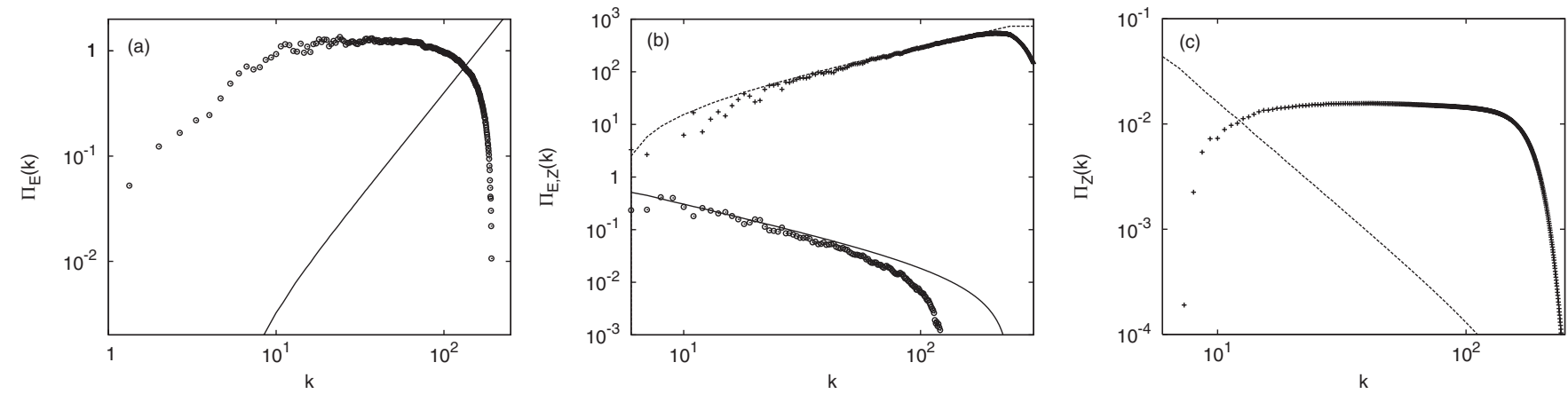

FIG. 3. The energy flux $\Pi_{E}$ (circles), the enstrophy flux $\Pi_{Z}$ (crosses), the energy input $I_{E}$ (thin line) and the enstrophy input $I_{Z}$ (dashed line) are shown for different values of $\varepsilon$. (a) $\varepsilon=1$; (b) $\varepsilon=2.5$; (c) $\varepsilon=4$. The energy input is (see text) $I_{E}(k)=I_{E}(k, 0)$ for $\varepsilon<2$ and as $I_{E}(k)=I_{E}(k, \infty)$ for $\varepsilon>2$, while the enstrophy input is $I_{Z}(k)=I_{Z}(k, 0)$ for $\varepsilon<3$ and as $I_{Z}(k)=I_{Z}(k, \infty)$ for $\varepsilon>3$. Parameter as in Fig. 2. 
gent in the absence of cutoffs. In this situation, the steady state is attained when the energy and enstrophy fluxes balance scale by scale the corresponding inputs [Fig. 3(b)]. In this $\varepsilon$ range the energy spectrum scales in agreement with the exponent which can be extrapolated but no longer fully justified using the RG. Finally, for $\varepsilon>3$ both the energy and enstrophy inputs become infrared dominated. In such a case the right-hand sides of Eq. (6) and of the analogous equation for the vorticity correlation admit a regular Taylor expansion for $m x \ll 1[19,29]$. The hypotheses (i), (ii), (iii) at $\xi_{0}=0$ thus recover $S_{3}(x) \propto x^{3}$, i.e., Kraichnan's scaling for the direct enstrophy cascade in agreement with our numerical observations [Fig. 3(c)].

In conclusion, our numerics shows that the scaling properties of 2D turbulence stirred by power-law forcing can be well explained in the framework of Kraichnan's phenomenological theory for all values of the Hölder exponent of the forcing spectrum. RG scaling prediction is not observed in the range where it was supposed to appear. It seems to us that this is not trivially a consequence of low Reynolds number $\left[O\left(\varepsilon^{1 / 2}\right)\right.$ for $F_{0}=O(\varepsilon)$ see discussion in section 9.6 .4 of [1] ] entailed by perturbative expansion.

Instead scaling seems to be related to the convolution of the response field with the forcing kernel in the SchwingerDyson equation $[6,28]$ giving rise to a nonlocal scaling field of dimension $d_{O}=-1 / 3$ independently of $\varepsilon<2$. This point, however, deserves more theoretical inquiry which is of special interest because power-law stirring in 2D appears to generate fully developed turbulence already at $\varepsilon=0$ in sharp contrast with the 3D case.

We are pleased to thank J. Honkonen, A. Kupiainen, and P. Olla for numerous friendly and enlightening discussions. Numerical simulations have been performed on the "Turbofarm" cluster at the INFN computing center in Torino and on CLX at CINECA. A. M. was supported by COFIN 2006 No. N.2005027808 and by CINFAI consortium, P.M.-G. by the center of excellence "Geometric Analysis and Mathematical Physics" of the Academy of Finland and by FP5 EU Network Contract No. HPRN-CT2002-00300.

*mazzino@fisica.unige.it

"paolo.muratore-ginanneschi@helsinki.fi

stefano.musacchio@gmail.com

[1] U. Frisch, Turbulence (Cambridge University Press, Cambridge, U.K., 1995).

[2] K. R. Sreenivasan and R. A. Antonia, Annu. Rev. Fluid Mech. 29, 435 (1997).

[3] A. N. Kolmogorov, Dokl. Akad. Nauk SSSR 32, 19 (1941).

[4] D. Forster, D. R. Nelson, and M. J. Stephen, Phys. Rev. A 16, 732 (1977).

[5] C. DeDominicis and P. C. Martin, Phys. Rev. A 19, 419 (1979).
[6] L. Ts. Adzhemyan, A. V. Antonov, and A. N. Vasilév, The Field Theoretic Renormalization Group in Fully Developed Turbulence (Gordon and Breach, Amsterdam, 1999).

[7] A. Sain, Manu, and R. Pandit, Phys. Rev. Lett. 81, 4377 (1998).

[8] L. Biferale, A. S. Lanotte, and F. Toschi, Phys. Rev. Lett. 92, 094503 (2004).

[9] L. Biferale, M. Cencini, A. S. Lanotte, M. Sbragaglia, and F. Toschi, New J. Phys. 6, 37 (2004).

[10] R. Salmon, Geophysical Fluid Dynamics (Oxford University Press, New York, 1998).

[11] O.A. Ladyzhenskaya, The Mathematical Theory of Viscous Incompressible Flow (Gordon and Breach, New York, 1969).

[12] S. Kuksin and A. Shirikyan, Commun. Math. Phys. 213, 291 (2000).

[13] J. Bricmont, A. Kupiainen, and R. Lefevere, J. Stat. Phys. 100, 743 (2000).Commun. Math. Phys. 224, 65 (2001); 230, 87 (2002).

[14] W. E, J. C. Mattingly, and Y. G. Sinai, Commun. Math. Phys. 224, 83 (2001).

[15] M. Hairer and J.C. Mattingly, Ann. Math. 164, 993 (2006).

[16] R. Kraichnan, Phys. Fluids 10, 1417 (1967).

[17] G. K. Batchelor, Phys. Fluids 12, II-233 (1969).

[18] R. Kraichnan and D. Montgomery, Rep. Prog. Phys. 43, 547 (1980).

[19] D. Bernard, Phys. Rev. E 60, 6184 (1999); arXiv:condmat/0007106.

[20] H. Kellay and W. I. Goldburg, Rep. Prog. Phys. 65, 845 (2002).

[21] P. Tabeling, Phys. Rep. 362, 1 (2002).

[22] G. Boffetta, A. Cenedese, S. Espa, and S. Musacchio, Europhys. Lett. 71, 590 (2005).

[23] M. K. Rivera, P. Vorobieff, and R. E. Ecke, Phys. Rev. Lett. 81, 1417 (1998); Phys. Fluids 11, 2167 (1999).

[24] G. Boffetta, J. Fluid Mech. 589, 253 (2007).

[25] P. Olla, Phys. Rev. Lett. 67, 2465 (1991).

[26] J. Honkonen, Phys. Rev. E 58, 4532 (1998); J. Honkonen, Yu. S. Kabrits, and M. V. Kompaniets, arXiv:nlin/0201025.

[27] L. Ts. Adzhemyan, J. Honkonen, M. V. Kompaniets, and A. N. Vasilév, Phys. Rev. E 71, 036305 (2005).

[28] J. Zinn-Justin, Quantum Field Theory and Critical Phenomena (Clarendon Press, Oxford, 2002), 4th ed.

[29] D. Bernard, Europhys. Lett. 50, 333 (2000).

[30] E. Lindborg, Phys. Fluids 11, 510 (1999).

[31] C. Canuto, M. Hussaini, A. Quarteroni, and T. Zang, Spectral Methods in Fluid Dynamics (Springer-Verlag, New York, 1988).

[32] K. Nam, E. Ott, T. M. Antonsen, Jr., and P. N. Guzdar, Phys. Rev. Lett. 84, 5134 (2000).

[33] Y. K. Tsang, E. Ott, T. M. Antonsen, and P. N. Guzdar, Phys. Rev. E 71, 066313 (2005).

[34] This is equivalent to a Pauli-Villars regularization, the use whereof is well justified in RG calculations; see, e.g., [26,28].

[35] G. Boffetta, A. Celani, S. Musacchio, and M. Vergassola, Phys. Rev. E 66, 026304 (2002).

[36] E. Lindborg and K. Alvelius, Phys. Fluids 12, 945 (2000). 